\title{
FOUR (OR SO) NEW FINE-TUNING ARGUMENTS
}

\section{LYDIA MCGREW}

\begin{abstract}
Both proponents and opponents of the argument for the deliberate fine-tuning, by an intelligent agent, of the fundamental constants of the universe have accepted certain assumptions about how the argument will go. These include both treating the fine-tuning of the constants as constitutive of the nature of the universe itself and conditioning on the fact that the constants actually do fall into the life-permitting range, rather than on the narrowness of the range. It is also generally assumed that the fine-tuning argument should precede biological arguments for design from, e.g., the origin of life. I suggest four new arguments, two of which are different orderings of the same data. Each of these abandons one or more of the common assumptions about how the finetuning argument should go, and they provide new possibilities for answering or avoiding objections to the fine-tuning argument.
\end{abstract}

\section{INTRODUCTION}

One of the knottier criticisms of the fine-tuning argument (FTA) has been that of McGrew, McGrew, and Vestrup (2003, hereafter MMV) based on the possibility of infinite ranges for universal constant values. MMV argue that, if the range of the possible values that some constant of physics could take is infinite, and if there is no rational way to treat some of these values as more probable than others for purposes of the FTA, it is impossible for the proponent of the fine-tuning argument rationally to assign a low probability that that constant would fall within the life-permitting range given no design, because the relevant probability is strictly inscrutable. They base this conclusion on the fact that an equiprobability distribution across an infinite number of possibilities would violate the probabilistic requirement of countable additivity. In short, their argument is that infinity should not be treated in probabilistic terms as akin to 'very, very large'. On this argument, the likelihood 
comparison between the probability of a life-permitting universe given design and its probability on no design is simply impossible to make, and the FTA fails.

The four new fine-tuning (or fine-tuning-type) arguments given here may have remaining problems, but three of them appear to be in the clear as far as the specific MMV objection, and the fourth (here, argument \#2), though it bears the greatest similarity to the original, classic, FTA, is sufficiently different that there is at least some reason to hope that it escapes the MMV objection. The conditional ordering suggested for argument \#2 has an additional attraction - the fact that it appears to render moot the long-running discussion of observer selection effects.

Not all of these arguments are independent of each other. In particular, arguments \#3 and \#4 are actually two different ways of incorporating the same evidence - namely, evidence typically used in the biological design argument. In one of these, we incorporate that evidence before considering the specific evidence of the FTA, and in the other we incorporate it afterwards. ${ }^{1}$

Proponents of fine-tuning should be interested in the possibility of having more strings to their bow. And opponents and proponents alike should be interested in the probabilistic results of greater argumentative flexibility when we abandon some long-held assumptions about the way fine-tuning arguments work.

\section{ARGUMENT \#1: COSMOLOGICAL CONSTANT FINE-TUNING AS AN IN-WORLD EVENT}

Robin Collins (2009) discusses the fine-tuning of the cosmological constant at some length. Collins specifically describes the various contributions to the vacuum energy which, he says, are 'far in excess of the maximum life-permitting amount' (Collins 2009: 215). Here is how Collins describes the contribution of the Higgs field:

The first contribution we shall consider arises from the Higgs field postulated as part of the widely accepted Weinberg-Salem-Glashow electroweak theory. According to this theory, the electromagnetic force and the weak force acted as one force prior to symmetry breaking of the Higgs field in the very early universe when temperatures were still

${ }^{1}$ A reasoner who rejects argument \#2 as vitiated by the MMV objection will likely consider the distinction between arguments \#3 and \#4 to be trivial. 
extremely high. Before symmetry breaking, the vacuum energy of the Higgs field had its maximum value $\mathrm{V}_{0}$. This value was approximately $10^{53}$ [times the maximum vacuum energy density compatible with the existence of life]. After symmetry breaking, the Higgs field fell into some local minimum of its possible energy density, a minimum which theoretically could be anywhere from zero to $10^{53}$ [times the maximum vacuum energy density compatible with the existence of life] [...] (Collins 2009: 216).

Collins has something similar to say about the contribution of the inflaton field postulated by inflationary cosmology.

Inflationary universe models hypothesize that the inflaton field had an enormously high energy density in the first $10^{-35}$ to $10^{-37}$ seconds of our universe, resulting in an effective cosmological constant that caused space to expand [...]. By around $10^{-35}$ seconds or so, however, the value of the inflaton field fell to a relatively small value corresponding to a local minimum of its energy. Now, in order to start inflation, the initial energy density of the inflaton field, $\mathrm{D}_{\mathrm{i}}$, must have been enormously larger than [the maximum vacuum energy density compatible with the existence of life] [...]. The fact that the effective cosmological constant after inflation is less than [the maximum vacuum energy density compatible with the existence of life] requires an enormous degree of fine-tuning, for the same reason as the Higgs field mentioned [...] (Collins 2009: 217).

What is immediately noticeable about both of these accounts of the finetuning of contributions to the vacuum energy is that the descriptions give the distinct impression that these fine-tunings are in-universe events. From a layman's perspective, the picture here shows the contributions of both the Higgs field and the inflaton field as having exceedingly high values in the almost unimaginably early universe and then, for no apparent reason, falling suddenly to within an exceedingly narrow range as compared to those initial values - the range necessary for the universe to be life-permitting.

The entire MMV objection turns on the very different concept of fine-tuning as taking place pre-universally and across an infinite number of possible values of some constant, which values must be regarded as equiprobable if we assign probabilities to them at all, where the value selected will be partially constitutive, from the outset, of the universe that comes into existence. In-world events are an entirely different matter. While it may be difficult to come up with a principled probability, 
conditional on there being no design involved, for some in-world event, it does not at least prima facie have to involve anything like laying down a flat probability distribution over an infinite number of possibilities. In this case, the initial value that the fields actually did have provides at least some grip on the nature of the actual universe against the background of which the sudden fall to a local minimum took place and the probability of particular values. It is that fall in value that requires explanation, just as some other in-universe event - say, a car's sudden drop in speed and its stopping just a few inches away from a child in the road - which requires an explanation.

Although Collins devotes much space (pp. 240-251) to arguing for finite comparison ranges as one approach to answering the MMV objection, he never takes the in-universe event approach to any of his fine-tuning examples. He prefers to argue for an 'epistemically illuminated range' - the range about which scientists can make predictions given their current theories about our universe - as a relevant finite comparison range in order to avoid the problem of infinite ranges. But someone who accepts the MMV objection would understandably reply that the mere fact that our theories do not tell us what would happen if the values of certain numbers fell outside of this 'illuminated range' does not mean that possible values outside of that range do not exist and hence can be conveniently ignored. ${ }^{2}$ Indeed, Collins himself admits that outside of the epistemically illuminated range there might be more ways for a universe to be life-permitting (pp. 246, 248). In all of this, however, fine-tuning is being treated as constitutive of a universe rather than as an in-universe event with the other properties of the actual universe as a background. It seems plausible that Collins does not treat the fine-tuning of the cosmological constant as an in-universe event because he wants to discuss the fine-tuning of another contribution to the vacuum energy, the contribution of the zero-point energies of the fields associated with forces and elementary particles (p. 217). For this aspect of the fine-tuning of the constant, Collins has to find a comparison range in the Plank energy scale, which is, in fact, something very much like the epistemically illuminated region - the point at which quantum field theory breaks down and is no longer applicable. Since that fine-tuning of the cosmological constant apparently cannot be treated as an in-universe event, Collins presumably prefers not to emphasize the possibility of treating other aspects of the

${ }^{2}$ MMV (2003: 206) do make this reply in response to John Leslie. 
cosmological constant's fine-tuning as in-universe events.

For those who do not find the illuminated region response to MMV convincing but who remain interested in seeing what can be done with some variety of fine-tuning argument, this motive will not be compelling. If there are some aspects of the cosmological constant fine-tuning that appear to be immune from the MMV objection in the first place, these are worth special attention.

\section{ARGUMENT \#2: CONDITIONING ON THE NARROWNESS OF THE LIFE-PERMITTING RANGE}

The MMV objection presupposes that what is conditioned on in the FTA is the fact that the values of various constants do fall within the range required for the universe to be life-permitting. This is the classic form of the argument.

Temporally, of course, actual human subjects know from the time they are able to reflect on the matter that, whatever the necessary conditions are for life to exist in the universe, those conditions are satisfied. People know that life exists long before they learn (if they ever do learn) that there are such things as 'universal constants of physics' or that there is such a thing as the 'apparent fine-tuning' of such constants. Yet it seems that when a person is presented with the fine-tuning argument he has learned something new. The usual structure of the fine-tuning argument therefore corresponds to a hypothetical deletion of the proposition, 'The constants of the universe fall into the life-permitting range' from one's body of knowledge. Ipso facto, this is a deletion of the fact that life exists. (See Monton 2006: 415-17.)

Suppose that, instead, we structured the argument like this: Have as background evidence the fact that life exists and that, therefore, the necessary conditions for life to exist and for the universe to be lifepermitting (whatever those are) are satisfied. Then condition on what we actually learn when first presented with the fine-tuning argument namely, that specific universal constants must fall within a narrow range, i.e., that their falling within this narrow range is a necessary condition for the universe to be life-permitting. Call this new evidence $\mathrm{N}$, or, more precisely, $\mathrm{N}_{1}-\mathrm{N}_{\mathrm{n}}$ for some $\mathrm{n}$ pieces of alleged fine-tuning evidence. ${ }^{3}$

${ }^{3}$ This argument is similar to the 'infrared bull's-eye' argument advanced by John Roberts (2012). One difference is that it is unclear that Roberts is putting as much 
What advantage might this ordering have over the classic ordering as far as avoiding the MMV objection? One possible advantage is this: In the classic ordering, the proponent of the FTA puts $\mathrm{N}$ into background evidence and then considers instead L - namely, that the universe is indeed life-permitting, which, since we have $\mathrm{N}$ in background, entails that the constants fall into the narrow life-permitting range. The proponent of the classic FTA needs to show that $\mathrm{P}(\mathrm{L} \mid \mathrm{D})>\mathrm{P}(\mathrm{L} \mid \sim \mathrm{D})$ - that is, that the probability that the constants (or whatever specific constant is in question) fall within the life-permitting range is greater given that an intelligent agent selected the constants than given that no intelligent agent did so. The MMV objection centres on the right-hand probability $\mathrm{P}(\mathrm{L} \mid \sim \mathrm{D})$ - alleging that it is inscrutable because of the problem of infinite possible ranges and equiprobability.

If instead we take L to be in the background and condition on $\mathrm{N}$, we are arguing that it is more probable that we would find the lifepermitting range to be narrow given both $\mathrm{L}$ and Design than given $\mathrm{L}$ and no Design. We are arguing that $\mathrm{P}(\mathrm{N} \mid \mathrm{D} \& \mathrm{~L})>\mathrm{P}(\mathrm{N} \mid \sim \mathrm{D} \& \mathrm{~L})$. But in arguing for this probabilistic inequality we need not obtain either probability by comparing the life-permitting range to an infinite range of possible constant values. Rather, we are saying that we would expect more strongly to find, given $\mathrm{L}$ and $\sim \mathrm{D}$, that the life-permitting range was 'not narrow', where 'not narrow' could include any much larger but finite life-permitting range.

One can argue for this inequality in something like the following way: If some powerful designer set up the necessary conditions for the existence of life, including whatever universal constant values were required for that purpose, and life exists, then it is not particularly surprising that some universal constants have a narrow life-permitting range. The powerful designer would be quite capable of seeing to it that they fell within such a life-permitting range despite its narrowness. If, however, the universe somehow came into existence through a nonintelligent process, and life exists, we should expect more strongly to find that it is easy for the universe to be life-permitting, that things did not have to be 'just so' in order for life in the universe to be possible. Hence, we would on this hypothesis more strongly expect that the range

emphasis on the narrowness of the required range or 'target', as it is in his version. My argument \#2 was conceived independently of his and this paper drafted before his became available. 
of life-permitting values for these constants would be very large - i.e., not narrow.

This new casting of the FTA, despite its reversal of background and evidence, is not very unlike the original FTA, and for that reason a proponent of the MMV criticism could raise the most objections to it. First, there is the definition of 'narrow'. Collins, for example, sometimes uses definitions of 'narrow' that are tightly tied to his notion of the 'illuminated region' that he proposes as a finite comparison range for probabilistic purposes (Collins 2009: 244). If, as I am assuming here, the proponent of the MMV objection is unconvinced by Collins's argument for using the finite 'illuminated region' to generate a manageable probability in the classic FTA, it is difficult to see why the proponent would allow the same finite ranges to generate a concept of 'narrow' for this version.

The re-casting of the FTA, if it is to avoid the MMV objection, will have to rely not on Collins's illuminated finite ranges to supply a comparison in order to define 'narrow' but rather on examples for which a qualitative and intuitive notion of 'narrowness' appears to be sufficient. For example, consider the example of the relative fine-tuning of the force strengths of gravity and the electromagnetic force. The electromagnetic force is roughly $10^{39}$ times stronger than gravity. According to Dean Overman (1997: 134-5), if the relation (which it seems best to understand as a ratio) between these two forces were changed by one part in $10^{40}$, all stars would be either red dwarfs or blue giants, and life as we know it (dependent on a star like our sun) would not be possible.

Another example would be this one, from Collins:

One of these is the fine-tuning of gravity relative to the density of massenergy in the early universe and other factors determining the expansion rate of the Big Bang - such as the value of the Hubble constant and the value of the cosmological constant. Holding these other parameters constant, if the strength of gravity were smaller or larger by an estimated one part in $10^{60}$ of its current value, the universe would have either exploded too quickly for galaxies and stars to form, or collapsed back on itself too quickly for life to evolve. (Collins 2009: 215)

In a footnote (p. 215, n. 10) Collins notes that this same point can be expressed by saying that 'the density of matter at the Plank time [...] must have been tuned to one part in $10^{60}$ of the so-called critical density'. These seem to be examples in which we can simply say in a qualitative sense 
that the life-permitting range of the constants in question, or of their ratios, is narrow.

Moreover, MMV do not appear to question the proposition that the life-permitting range of the constants is narrow. In fact, they appear willing to grant it:

[L] et us grant that there is a plausible convention we may adopt as to the line of demarcation between life-friendly and life-unfriendly universes, and that this convention will give us a range, perhaps even a narrow one, within which each variable will have to fall in order for the universe to be life-friendly. (2003: 201)

More interesting still, the MMV rejection of the 'coarse-tuning argument' (CTA) implies that they grant at least a meaning to the concept of a narrow life-permitting range. The CTA, originally developed by MMV as an attempted reductio for a certain construal of probability in the FTA, involves comparing the ratio of the life-permitting range to an infinite range. Since any finite range has measure zero on an infinite range, the mere finiteness, not the narrowness, of the life-permitting range carries the entire strength of the argument. An acceptance of this argument has what MMV call the 'unhappy consequence' that a requirement for the constants to fall within any finite range, even one within a 'few billion orders of magnitude of our values' (in other words, a non-narrow finite range) would have equal force as an argument for design to the force of the FTA. (2003: 204)

In a later symposium McGrew and McGrew reject the CTA as obviously forceless (more so even than the FTA) precisely because it abandons the importance of a narrow range.

[T] he role of physics in the CTA is drastically attenuated; physical considerations do no more than indicate that the life-friendly [probabilistic] region [...] is finite. But all of the excitement about the FTA has centered on the alleged narrowness of the life-friendly regions, and even Collins's own comparison classes are derived by painstaking attention to physical considerations. We doubt that anyone would have considered the CTA to be even a possible argument for design were it not for the objections that have been raised against the FTA. (McGrew and McGrew 2005: 433)

Despite the term 'alleged' before 'narrowness', it seems fair to say that this argument for rejecting the CTA would lose much of its force if the 
concept of a narrow life-permitting region as used in the FTA were literally meaningless.

Another, and more telling, objection that a proponent of the MMV position might raise concerns the argument for the likelihood inequality where $\mathrm{N}$ is the evidence. ${ }^{4}$ Consider the brief version of that argument above. What does it mean to say that we would expect it to be easy, or easier (as opposed, presumably, to difficult) for the universe to be lifepermitting given no design? And how can we defend that statement? One is tempted to give a simple probabilistic modelling, and therein lies the difficulty. If one attempts to model the notion of 'easiness' or 'difficulty' of the production of a life-permitting universe by non-design, an obvious way to do so is by means of a stochastic process, such as the random selection of balls from an urn. If we get a black ball from an urn and are asked to guess whether the urn contains, say, one black ball out of a million or 500,000 black balls out of a million, the fact that we got a black ball supports the latter hypothesis over the former. This model, though, will not do if one takes the MMV objection seriously in the first place. For in such an ordinary model, the total number of balls over which the stochastic process ranges is finite, though it could be very large, and though our number of draws may be in principle infinite. When we think of the makeup of the population, however, we are thinking of the ratio of balls with the property of interest (being black) to the total number of balls. If the total number of balls were infinite and we were asked to imagine the chance process as 'ranging randomly' over such an infinite space, such a ratio would not, according to the MMV objection, yield a meaningful probability.

In other words, one obvious casting of the argument for the likelihood inequality even in this version of the argument takes us back to saying that the probability of a life-permitting universe on $\sim D$ is low if the range is narrow and would be higher if the range were larger. Of course, the proponent of the MMV objection will not allow any such argument. If we could say that the probability of a life-permitting universe is low if the range is narrow, we could use the classic FTA in the first place!

It is difficult to say whether the proponent of the MMV objection will be able to be convinced, therefore, of a likelihood inequality for $\mathrm{N}$ favourable to $\mathrm{D}$. The best approach to trying to convince him is not to use the above model but rather to say simply that, all else being equal,

${ }^{4}$ I owe this ingenious and difficult objection to David Glass. 
it would be easier, given $\sim \mathrm{D}$, for the universal constants to fall within the life-permitting range if the range were large than if the range were narrow; hence, we should expect, given that the universe is life-permitting and given no design, to find that the range is not narrow. The ceteris paribus condition specifies that, if we compare a situation in which some nondesign process is generating the universe (whatever that would look like) and the life-permitting range is narrow to a situation in which the same process is generating the universe and the range is large, this does not involve any change in the total possible universes from which the process is choosing. It does not specify that this total possible range is either finite or infinite and does not, per se, require directly using the concept of low probability derived from comparing the life-permitting range to the total range of possibilities.

It is possible that the proponent of the MMV objection will still reject this version of the argument. ${ }^{5}$ If so, there remain (for answering that critic) the other versions discussed in this article. But there is one more point to be made before leaving argument \#2: Much philosophical argument has gone on about a different objection to the FTA - the objection based on an observer selection effect. Here is John Leslie's characterization:

Any intelligent living beings that there are can find themselves only where intelligent life is possible. (Leslie 1996: 128)

\section{Or, as Leslie quotes B. Carter,}

[W] hat we can expect to observe must be restricted by the conditions necessary for our presence as observers. (Leslie 1996: 128)

The idea, then, is that we should not be terribly surprised to observe that our universe is life-permitting, as a life-permitting universe is the only sort of universe we could observe anyway. Perhaps, for all we know, there have been many 'failed' (i.e., not life-permitting) universes generated (which we could not have observed), and ours just happened to be the one out of the many that was life-permitting.

\footnotetext{
${ }^{5}$ It seems more plausible that he would do so because MMV in their original article reject, given that the possible range is infinite, any attempt to take the ratio of the narrow range to the total possible range as indicative of probabilistic force. (MMV 2003: 204) The salient question is whether some notion of the ratio of the life-permitting range to the total range of possibilities is being smuggled in here under the heading of its being 'easier' or 'harder' for a non-design process to generate a life-permitting universe depending on the size of the life-permitting region.
} 
There are many things that can be (and have been) said in answer to this 'anthropic principle' and its concomitant invocation of an ensemble of 'failed' universes. (See McGrew, L. 2005.) One point that has not perhaps been sufficiently considered, and that relates indirectly to something discussed below (there, a Cartesian objection to the FTA), is that if one is a Cartesian dualist the entire anthropic objection may be moot; a life-permitting universe is not, on that view, a necessary condition for 'our' presence as observers, especially when 'we' is construed, as it must be in the observer selection effect objection, to refer to whoever is in fact observing the world. Disembodied beings could be observers of non-life-permitting universes, so perhaps we should be surprised at not observing a non-life-permitting universe after all.

The new FTA discussed in this section makes the observer selection effect objection to the FTA irrelevant in yet a different way. Even if one were to grant for the sake of the argument that we could not observe anything if life were impossible in our universe, it would not be impossible for us to observe $\sim \mathrm{N}$. If we put $\mathrm{L}$ into our background and concede that, indeed, we are here to observe whatever we do observe, it remains surprising that the life-permitting range is narrow. Why did it not turn out that the life-permitting range was large, that there is nothing special about it, that things did not have to be 'just so' for our universe to permit life? There is simply no observer selection effect in that observation at all. It seems that we could quite easily have observed $\sim N$. (This point was independently made by Roberts 2012.)

If one looks into the argument for the likelihood inequality - $\mathrm{P}(\mathrm{N} \mid \mathrm{D}$ \& $\mathrm{L})>\mathrm{P}(\mathrm{N} \mid \sim \mathrm{D} \& \mathrm{~L})$ - there still seems no place for the observer selection effect to get purchase. The argument for the likelihood inequality says that given $\sim \mathrm{D}$ (and a life-permitting universe) we should be somewhat surprised to find that $\mathrm{N}$, since it would have been easier for a non-design process to generate a life-permitting universe given $\sim \mathrm{N}$. An acknowledgement of this point is implicit in the very multiplication of universes which, together with an observer selection effect, is presented on the non-design side as an 'explanation' in response to the classic FTA. If we had observed $\sim \mathrm{N}$ in the first place, no such explanation as a series of multiple, botched universes, resulting eventually by sheer multiplication of chance resources in one life-permitting universe, would have been postulated. It is precisely the apparent difficulty of producing a life-permitting universe in a single 'run' of a non-design process that gives rise to the multiverse-plus-selection-effect theory. 
A person unmoved by the MMV objection may find this new version of the FTA attractive because it avoids the observer selection effect issue. ${ }^{6}$ It is the narrowness of the range that we are conditioning on, and we could easily have observed otherwise.

The idea of conditioning on $\mathrm{N}$ rather than on $\mathrm{L}$ can be applied to argument \#1 as well, and hence is available, for purposes of not having to worry about the observer selection effect response, to someone who rejects argument \#2 but is open to argument \#1.

\section{ARGUMENT \#3: THE BIOLOGICAL DESIGN ARGUMENT FIRST}

Suppose that you think that an argument for the design of some biological entities within this universe - for example, the argument from the origin of life - has significant force. It would be easy to assume that the fine-tuning argument must be made first and the biological design argument later. It would also be easy to assume that the biological design argument (BDA) has no particular relevance for the specific conclusion of the FTA - that a powerful intelligent entity selected the values of some or all of the fundamental constants of the universe.

Both assumptions are mistaken. The structure of argument $\# 2$ helps us to see why the first assumption is mistaken. If we do the FTA by conditioning not on the fact that the universe is life-permitting but rather on the narrowness of the life-permitting range, we are not required to 'subtract out' all arguments that entail (either by their evidence or by their conclusions) that the universe is life-permitting. Therefore, we are not absolutely required to do the FTA before the BDA.

Bradley Monton (2006: 418-19) uses the apparent need to do a radical deletion of the fact that the universe is life-permitting to argue that some subjects might reasonably have a very low prior probability for theism when going into the FTA. For example, Monton points out that any argument from miracles takes as background information the fact that life exists. Any argument from the appearance of design of specific biological entities (Monton instances Paley's argument about the eye)

${ }^{6}$ A person who thinks this version does avoid the MMV objection has the avoidance of the observer selection effect issue as an additional reason for preferring the approach of conditioning on $\mathrm{N}$ rather than on L. Moreover, as we shall see in the next section, there are independent reasons why one might wish to consider the biological design argument before doing the FTA, and in that case one would have in background the fact that the universe is life-permitting before doing any version of the FTA. 
assumes that the universe is life-permitting. So, Monton argues, to the extent that the FTA is intended to be an argument for even a generic form of theism, the only arguments that can be made prior to it and can help to set a 'decent' or 'not very low' prior probability for theism before the FTA will be metaphysical arguments such as the cosmological argument or the ontological argument. ${ }^{7}$

The possibility of setting $\mathrm{L}$ as background gives us more options for ordering, but it's unclear that Monton's point is a sufficient reason for wanting to reverse the order and do a biological design argument first. After all, some proponents of the cosmological and ontological arguments would say that those arguments give a very high probability to theism, perhaps even probability 1, so this point of Monton's will not faze them.

But even aside from a theistic interpretation of the fine-tuning argument and the prior probability of theism, a successful BDA, or a BDA with some significant force, gives us reason to believe that a very powerful, intelligent entity or entities exist with an interest in bringing about biological life on earth. If that is true, then it becomes less improbable that this being, or a being of this type, would bring about the necessary conditions for life on earth. If the subject has the concept of fundamental constants that can take varying values, he can form the belief that a designer might have been around and motivated to do any fine-tuning of those values that happened to be necessary to make the universe life-permitting. In other words, the conclusion of the BDA e.g., that a powerful intelligent being was responsible for the origin of life on earth - raises the prior probability of the conclusion of the FTA - that an intelligent being selected the values of the fundamental universal constants. $^{8}$

We can see this point fairly readily in reverse: If a powerful being did take the trouble to select the fundamental constant values to make the universe life-permitting, this gives us some reason to believe that a being exists who is both capable of making life on earth and desires to bring

${ }^{7}$ Monton also says that the force of the argument from consciousness would have to be deleted. I disagree, since one's access to the existence of one's own consciousness is direct and does not depend on empirical premises about the existence of biological life.

${ }^{8}$ It need not even, strictly speaking, be the same being, though the positive relevance is stronger on the assumption of some connection (even a connection of planning or anticipation on the part of the being designing the fundamental constants) between the beings. 
about life on earth. To give a simple example, suppose that you were to find in the lounge of a college dormitory a fish tank containing water. This would give you some reason to think that the person or persons who took the trouble to provide these necessary conditions for keeping live fish in the lounge would eventually provide the fish (since fish do not arise spontaneously in fish tanks). This makes it fairly evident that, if the conclusion of the FTA is true, and if it turns out that living cells on the early earth, like fish in an aquarium, are highly unlikely to appear on their own by purely natural processes, we have some reason from the conclusion of the FTA alone to think that a powerful intelligent agent deliberately brought about life on earth. I will return to this point in the next section.

Positive relevance is symmetrical. If the conclusion of the FTA is positively relevant to the conclusion of the BDA, then the opposite relevance relation also holds: The conclusion of the $\mathrm{BDA}$ is positively relevant to the conclusion of the FTA. An intelligently provided fish tank gives us reason to expect fish. Intelligently provided fish give us reason to think that someone deliberately provided the (absolutely necessary) fish tank as well.

This point regarding the positive relevance between the conclusion of the BDA and the conclusion of the FTA is relevant to what we might call a Cartesian objection to the FTA. Suppose that consciousness is possible in non-biological entities. Suppose that there could be conscious black holes or conscious red dwarf stars. In that case, even if there exists a powerful designer (say, God), and even if this designer wanted to design the universe to be a certain way, do we have sufficient reason to believe that he would make the universe life-permitting? ${ }^{9}$ If the only reason for thinking that life might be special to a designer is the connection of complex life with consciousness and the idea that a designer would want to bring about other conscious, intelligent beings somewhat like himself, a Cartesian could argue that he would be just as likely to implant consciousness in black holes or other 'boring' forms of matter and would have no special interest whatsoever in complex life of the sort that requires universal fine-tuning as a necessary condition.

\footnotetext{
${ }^{9}$ As noted above, if one takes seriously the possibility of conscious black holes and the like, one cannot then make use of the observer selection effect as a putative weakness of the FTA. So one cannot consistently press both a Cartesian objection to the FTA and an observer selection effect objection.
} 
This objection can be answered in a number of ways. One important point is that the advocate of the FTA merely needs a likelihood inequality. He needs to show that the probability of $\mathrm{L}$ (or of $\mathrm{N}$, depending on which direction he is doing the argument) is greater on $\mathrm{D}$ than on $\sim \mathrm{D}$, not that it is positively high on D. Still, it could be quite useful for the FTA proponent to have already in hand other evidence directly supporting the existence of at least one powerful designer who does want to have complex life in the universe and hence, presumably, wants a lifepermitting universe.

\section{ARGUMENT \#4: BIOLOGICAL DESIGN ARGUMENT SECOND}

This argument represents another ordering one might use for taking into account the impact of the BDA on the conclusion of the FTA. As noted at the outset, it is therefore not independent of argument \#3 but is rather a different use of the BDA argument in relation to the FTA. Some reasoners might find it conceptually preferable or cleaner to do the FTA (with whatever force it has) first and then to layer on top of that any further evidence for in-world design of biological entities.

In this argument ordering, then, one has already taken into account both $\mathrm{L}$ and $\mathrm{N}$. We are envisaging a situation in which the reasoner already knows that the universe is life-permitting and indeed contains life (of some kind) and that the life-permitting range for the values of fundamental universal constants is narrow. The reasoner has not yet taken into account the details of, say, the organization of the cell and the challenge these pose for abiogenesis theories. ${ }^{10}$ Let us suppose that, when he does so, this version or aspect of the BDA provides some support worth noting for the conclusion that life on earth was intelligently designed.

The point to be noted here, which we have already discussed to some extent while looking at argument \#3, is that this conclusion gives further support of its own for the conclusion of the FTA - that the values of the fundamental constants of the universe themselves were selected by

10 There is a resemblance here between the BDA and conditioning on $\mathrm{N}$ rather than $\mathrm{L}$ for the FTA. When it comes to biology, people know in some sense that life exists long before they learn anything about the structure of DNA or anything else about detailed cellular structure and the fact that this microstructure had to be 'just so' in order for life to begin on earth. It is that new information that they actually condition on when they study the details of a BDA based on the origin of life. 
a powerful intelligent agent. If an intelligent designer took the trouble to make intelligent life on earth, this gives us some reason to think that a powerful intelligent designer also would be motivated, if it were necessary, to design the universe itself to be a life-permitting 'habitat'. Conversely, if a designer took the trouble to provide a life-permitting universe, he or perhaps others like him were not indifferent to whether or not life actually came into existence in the universe and, if necessary, would and could provide further necessary conditions for the actual appearance of life on earth. The conclusion of the BDA is positively relevant to the conclusion of the FTA and vice versa, whichever order one considers them in. ${ }^{11}$

This point is relevant to complaints such as that of Elliott Sober (2007) to the effect that advocates of a design hypothesis in biology have absolutely no idea what motives or goals a designer might have and hence no purchase for making a probabilistic comparison between the probability of the biological evidence given design and given no design. As in the case of the hypothetical Cartesian objection to the FTA considered above, there are more answers than one to Sober's complaint. (See McGrew, L. 2004.) But as with argument \#3, so here: Whichever order one chooses for conditioning on the evidence used in the BDA and the FTA, to the extent that either argument has any force on its own, it provides evidence concerning precisely the 'goals and interests' question Sober is raising about a powerful designer and the existence of complex life on earth.

Moreover, a reasoner who considers the probabilistic FTA to be forceless or nearly forceless, e.g., a reasoner convinced that the MMV

${ }^{11}$ Throughout this discussion I am treating the FTA, concerning the fundamental laws and constants, and the BDA, concerning life itself or the details of some aspect of living creatures, to be separate arguments based on separate sets of evidence. Richard Swinburne (2004: 172, 189) is, unfortunately, rather unclear on this point. Although he acknowledges that the fundamental laws and constants mentioned in the FTA are necessary but not sufficient for the actual existence of life, he also says that they make the existence of life in the universe probable, which is, to put it mildly, a contentious claim and forms no part of the FTA. The FTA concerns merely life-permitting laws and constants, not (for purposes of the argument) life-producing laws and constants. Swinburne goes so far as to assert (p. 189) that, from the existence of human and animal bodies, there will be an argument to the existence of God that has any strength 'via fine-tuning' only if fine-tuning makes it significantly probable that such bodies will develop, which is an extremely puzzling statement, since no version of the FTA involves conditioning on the actual existence of human and animal bodies. 
objection is insuperable for both the classic FTA and for the somewhat similar argument \#2 above, can grant the positive relevance of the BDA to the FTA conclusion. If the evidence for the conclusion of the BDA is any good in itself (obviously, a separate and highly contentious question), it provides some evidence, since we are assuming that $\mathrm{N}$ is also already in one's background, for the conclusion of the FTA, that a powerful intelligent agent 'tuned' the fundamental constants and laws of the universe to be life-permitting, independent of the success of a classic, probabilistic FTA. Therefore, even a reasoner who does not draw the conclusion of the FTA from the FTA evidence by itself may draw this conclusion later after conditioning both on $\mathrm{N}$ and on the evidence used in the BDA.

\section{A WORD ON EXPLANATION}

The various new fine-tuning arguments examined here are possible in no small part because a piece of evidence can support an hypothesis even if the hypothesis does not explain the evidence. It is attractive to think of evidence-hypothesis connections as explanatory and to think of Bayesian inference as a probabilistic parsing out of inference to the best explanation. So it often is, but it need not always be so. Consider the example of an archer and a target. Suppose that we do not know the size of the target but are told that Susan has hit it. If we then discover the target to be very small, we have some reason to believe that Susan is a skilful archer. This inference - from the smallness of the target that has been hit to the skill of the archer - is not an explanatory inference. The archer's skill does not explain the smallness of the target. Rather, having already been told that the archer hit the target, we know that this will be easier for an unskilled archer if the target is very large than if it is very small. Hence, we expect the smallness of the target more strongly on the hypothesis that the archer was skilled, and the discovery that the target was small supports the hypothesis of a skilled archer. Somewhere buried in all of this we can find an explanatory move - namely, in the idea that the skill of the archer explains the fact that the archer hit the target even though the target is small. But if we discover that the archer hit the target first and that the target is small second, the probabilistic inference that the archer was skilled from the smallness of the target is not in itself an explanatory inference. This argument, of course, is very roughly analogous to argument \#2, above. 
I have emphasized repeatedly that the conclusions of the FTA and BDA are positively relevant to each other, but neither of them explains the other. The conclusion of the BDA is relevant to the conclusion of the FTA for more indirect reasons - roughly, because they both point either to a powerful designer or to a set of powerful designers who desired and/or intended to bring about the existence of life in the universe. It is the action of such a designer or designers that is asserted in both conclusions. Hence, arguments \#3 and \#4 are not explanatory in the usual sense, either.

Of all of the new fine-tuning arguments considered here, the only explanatory one is \#1 - the argument from the fine-tuning of the cosmological constant, construed as an in-world event. There, the deliberate action of a powerful intelligent agent is being treated as the explanation for the fact that the relevant contributions to the vacuum energy suddenly fell to within the narrow, life-permitting range.

We increase our flexibility in making and understanding arguments if we do not tie ourselves too tightly to the explanatory model - a point that can be helpful not only to proponents of fine-tuning and other design arguments but also to those making and considering evidence in other scientific areas.

\section{SOME SAMPLE ORDERINGS}

In the course of this discussion we have already seen, in outline, some ways that various design and fine-tuning arguments could be ordered, but it may be helpful to note in more detail how they, and their component parts, could go. The possibility of separating L in its generic form from empirical details about what is required for the existence of life or for a life-permitting universe, which the subject will usually acquire much later, plays an important role in permitting a variety of possible design argument orderings.

Example 1: The subject, who never considered the MMV objection to have any serious force and is unfazed by the selection-effect-plusmultiple-universes objection, first runs a classic FTA. That is, he places $\mathrm{N}$ into background knowledge, subtracts $\mathrm{L}$ from his knowledge, and conditions on $\mathrm{L}$. ( $\mathrm{L}$ and $\mathrm{N}$ taken together entail that the universal constants do fall into the life permitting range.) Since he was not bothered by the MMV objection in the first place, he makes no special distinction between the cosmological constant fine-tuning as an in-world event and 
any other fine-tuning. He then conditions on evidence for some version of the BDA and, having read this paper, recognizes that the BDA provides evidence not simply for its own conclusion regarding, say, the origin of life but also provides additional evidence for the deliberate fine-tuning of the universal constants.

Example 2: This subject is much like the subject in example 1, except that he has been concerned about the observer selection effect objection. (He doesn't consider the MMV objection to be a problem.) He therefore first puts $\mathrm{L}$ in a generic form into his background evidence - namely, that life does exist in the universe and hence that whatever the necessary conditions are for life (not specifying these but including a generically life-permitting universe), they are satisfied. He runs the FTA by conditioning on $\mathrm{N}$ (rather than $\mathrm{L}$ ) in order not to have to worry about the observer selection effect and multiple universes. From there on he proceeds as does the reasoner in example 1.

Example 3: This subject has been bothered by the MMV objection. Though he doesn't necessarily consider the selection effect objection to be a problem, he is supremely bored by the vast literature on observer selection effects and multiple universes. He puts generic L into background. He then conditions on evidence for the BDA, which gives some support to the conclusion that life on earth was deliberately designed by an intelligent agent. He also recognizes that, if that is the case, that gives us some reason to think that a powerful intelligent agent would have designed the fundamental constants of the universe if necessary, so he has some reason to believe the conclusion of the FTA. It is also useful to realize, from the conclusion to the BDA, that there is less reason later to worry about the Cartesian objection (concerning nonliving intelligences) to the FTA. He next conditions on $\mathrm{N}_{1}$, concerning the narrowness of the life-permitting range for the cosmological constant and its apparently having fallen into that range in the very early universe. $\mathrm{He}$ construes its falling into the life-permitting range as an in-world event. He considers this to provide significant evidence for the conclusion of the FTA with regard to the cosmological constant. He then conditions on $\mathrm{N}_{2-\mathrm{i}}$, for other fundamental constants. All of these N's say that the conditions for a life-permitting universe had to be 'just so'. This subject considers that the N's strongly support the conclusion for the FTA - that is, that the probability of $\mathrm{N}$ given design is significantly greater than the probability of $\mathrm{N}$ given no design. This gives him additional, stronger support for the conclusion of the FTA. 
Example 4: This subject is somewhat similar to the subject in example 3, except that, even after examining the argument given here, he continues to think that an MMV-type objection is fatal both to the classic FTA and to argument \#2, above. He therefore casually places both $\mathrm{L}$ and $\mathrm{N}_{2-\mathrm{i}}$ into background evidence, though that does not raise, in his mind, the probability of the conclusion to the FTA. He then conditions on $\mathrm{N}_{1}$ for the cosmological constant as did the subject in example 3. This subject agrees that the apparent fine-tuning of the cosmological constant does raise the probability of the conclusion to the FTA. He follows this by conditioning on the evidence for the BDA, which, since he considers it to have force in favour of its own conclusion - that life on earth is significantly explained by the action of an intelligent designer - also raises the probability of the conclusion to the FTA. He, like the subject in example 3 , is quite pleased not to have to wade through any more articles on observer selection effects and multiple universes, not even in order to condition on the fine-tuning of the cosmological constant.

\section{CONCLUSION}

Suppose that you, the reader, do not think that either the FTA or the BDA has much force or is terribly interesting. Perhaps, especially when considering the BDA, you think that the evidence is exceedingly poor and that intelligent design theorists are charlatans attempting to fool an unwary and ignorant public. Perhaps you have some other objection to the FTA not addressed here.

Even so, the probabilistic considerations raised here have interest that transcends their direct application to these design arguments. Consider the issue of argumentative order. It is a natural assumption that we should layer our arguments causally, first presenting evidence concerning the origin of some necessary set of pre-conditions for some later development, then presenting evidence about how that later development actually happened. The new FTA arguments here show that it need not always be so and that sometimes we can gain epistemic enlightenment and get a probabilistic grip on a problem by reversing that order.

The issue of explanation has been discussed above in detail. It is useful to have Bayesian arguments for hypotheses that do not explain the evidence for them as a tool in our probabilistic toolkit. 
Finally, the possibility of assuming a generic notion of $\mathrm{L}$ - that the universe is evidently life-permitting (e.g., because life exists) - and then conditioning on further information showing how difficult it is for life to exist or for the universe to be life-permitting allows a more sophisticated understanding of deletion and conditioning than has previously been evident in design arguments. This understanding is relevant even to non-design causal inferences in science. We may know quite well in general terms that some biological system exists, but there is no need to 'subtract out' this generic knowledge when comparing, say, two different evolutionary models for its coming into existence. They can be compared for their virtues as explanations of the details of the system, details that turn out to be necessary for its existence and operation but that need not be brought into the picture insofar as the system is conceived in generic terms. $^{12}$

The probabilistic flexibility exemplified by these four (or so) new finetuning arguments is therefore to be recommended generally in science and in the philosophy of science. ${ }^{13}$

\section{BIBLIOGRAPHY}

Collins, R. 2009. 'The teleological argument: an exploration of the fine-tuning of the universe', in W. L. Craig and J. P. Moreland (eds), The Blackwell Companion to Natural Theology (West Sussex: Blackwell), pp. 202-281

Leslie, J. 1996. Universes (New York: Routledge)

McGrew, L. 2004. 'Testability, Likelihoods, and Design', Philo, 7 (1) (SpringSummer): 5-21

McGrew, L. 2005. 'Likelihoods, Multiple Universes, and Epistemic Context', Philosophia Christi, 7: 475-81

McGrew, T., McGrew, L., \& Vestrup, E. 2003. 'Probabilities and the Fine-Tuning Argument: A Sceptical View', in N. Manson (ed.), God and Design (New

12 When considering the evidence for and against various scenarios, such as varying evolutionary scenarios, for the origin of the mammalian eye, scientists make use of data collected using, inter alia, their own eyes. If we did a truly radical deletion of a proposition like, 'The mammalian eye reliably conveys information about the environment', we would find it difficult to gain access to most of our evidence about the visual mechanism. After all, that evidence itself rests on the tacit assumption that the investigating scientists' eyes are working properly. But scientists sense instinctively that such a radical deletion is not required; it is the underlying details of the visual mechanism, not the existence of some working visual mechanism or other, that we are treating as an explanandum.

${ }^{13}$ My thanks to David Glass for lengthy and challenging correspondence on the topics in this article which forced me to qualify my conclusions concerning Argument \#2. 
York: Routledge), pp. 200-208. Originally published in Mind, 110 (October, 2001): 1027-37. Pagination used throughout this article is from the 2003 printing.

McGrew, T. \& McGrew, L. 2005. 'On the Rational Reconstruction of the FineTuning Argument: Response to Collins and Pruss', Philosophia Christi, 7 (2): 425-443

Overman, D. 1997. A Case Against Accident and Self-Organization (Lanham, MD: Rowman \& Littlefield)

Roberts, J. 2012. 'Fine-tuning and the Infrared Bull's-Eye', Philosophical Studies, 160: $287-303$

Sober, E. 2007. 'The Design Argument' (expanded version, article originally published 2004), available at: http://sober.philosophy.wisc.edu/selectedpapers [Accessed 11/4/13]

Swinburne, R. 2004. The Existence of God, 2nd edition (Oxford: Oxford University Press) 